\title{
The Population Benefit of Radiotherapy for Malignant Brain Tumors: Local Control and Survival Estimates for Guideline-Based Use
}

\author{
Timothy Paul Hanna, MD, MSc, PhD, FRCPC ${ }^{\mathrm{a}, \mathrm{b}}$; Geoffrey Paul Delaney, MBBS, MD, PhD, FRANZCR ${ }^{\mathrm{a}}$; \\ and Michael Bernard Barton, MBBS, MD, FRANZCR ${ }^{a}$
}

\begin{abstract}
Objective: To estimate the population benefit of radiotherapy (RT) for primary malignant brain tumors if evidence-based guidelines were routinely followed. Methods: This study investigated 5-year local control (LC) and 2- and 5-year overall survival (OS) benefits. RT benefit was the absolute proportional benefit of RT alone over no RT for radical indications, and over surgery alone for adjuvant indications. Chemoradiotherapy (CRT) benefit was the absolute incremental benefit of concurrent chemotherapy and RT over RT alone. Decision tree models were adapted to define the incidence of each indication. Citation databases were systematically queried for the highest level of evidence defining indication benefits. Meta-analysis was performed if there were multiple sources of the same evidence level, and deterministic and probabilistic sensitivity analysis was also performed. Results: Among all patients with malignant brain tumors, $82 \%$ had indications for curative- or adjuvant-intent RT. The magnitude of benefit was based on level I or II evidence in $44 \%$ of all patients. A total of 25 relevant studies were used to quantify indication benefits. All RT benefit included in the model was irreplaceable. For malignant brain tumors, the estimated population benefit for RT alone was 9\% for 5-year LC (95\% Cl, 7\%-10\%), 9\% for 2-year OS (95\% Cl, 8\%-11\%), and 5\% for 5-year OS (95\% Cl, 4\%-5\%). The incremental benefit of CRT was $1 \%$ for 5 -year LC (95\% Cl, $0 \%-2 \%), 7 \%$ for 2 -year OS (95\% Cl, $4 \%-11 \%)$, and $3 \%$ for 5 -year OS $(95 \% \mathrm{Cl}, 1 \%-5 \%)$. The model was robust in sensitivity analysis. Conclusions: When optimally used, RT provides an important benefit for many patients with malignant brain tumors. The model provided a robust means for estimating the magnitude of this benefit.
\end{abstract}

J Natl Compr Canc Netw 2016;14(9):1111-1119

\section{Background}

Radiotherapy (RT) is vital to the management of primary malignant brain tumors. It has been estimated that $80 \%$ of patients with malignant brain tumors have an indication for RT because of superior local control (LC), survival, or palliative outcomes. ${ }^{1}$ The magnitude of the population benefit of RT, if optimally used according to guidelines, in patients with malignant brain tumors has not previously been estimated. This infor-

From ${ }^{a}$ Collaboration for Cancer Outcomes Research and Evaluation (CCORE), Ingham Institute, University of New South Wales, Australia; and bivision of Cancer Care and Epidemiology, Cancer Research Institute at Queen's University, Kingston, Ontario, Canada.

Submitted April 12, 2016; accepted for publication May 26, 2016.

The authors have disclosed that they have no financial interests, arrangements, affiliations, or commercial interests with the manufacturers of any products discussed in this article or their competitors. Dr. Hanna holds a research chair supported by the Ontario Institute for Cancer Research (OICR) through funding provided by the Government of Ontario (\#IA-035). There were no other sources of funding. OICR was not involved in the design and conduct of the study; collection, management, mation would be of use for economic analysis, planning, and priority setting. . $^{2,3}$

This study aimed to estimate the population overall survival (OS) and LC benefits of RT for malignant brain tumors. The distinct benefits of RT, separate from the benefits of other treatment modalities, are considered. The incremental benefit of radiosensitizing concurrent chemoradiation (CRT) over RT alone is described. This study used an evidence-based approach,

analysis, and or interpretation of the data; OICR was not involved in the preparation, review, or approval of the manuscript.

Author Contributions: Study concept and design: Hanna, Delaney, and Barton. Acquisition, analysis, or interpretation of data: Hanna, Delaney, and Barton. Drafting of manuscript: Hanna. Critical revision of the manuscript for important intellectual content: Hanna, Delaney, and Barton. Statistical analysis: Hanna. Administrative, technical, or material support: Hanna, Delaney, and Barton. Study supervision: Delaney and Barton.

Correspondence: Timothy Paul Hanna, MD, MSc, PhD, FRCPC, Division of Cancer Care and Epidemiology, Cancer Research Institute at Queen's University, 10 Stuart Street, 2nd Level, Kingston, Ontario K7L3N6, Canada. E-mail: thanna@kgh.kari.net 
Hanna et al

building on well-described whole-population RT demand models. ${ }^{1-4}$

\section{Methods}

\section{Definitions of Benefit}

End Points: This study investigated 5-year actuarial LC and 2- and 5-year OS benefits. The distinct LC and OS benefits of RT alone and concurrent CRT were considered. Palliative benefits were not investigated.

RT Benefit: RT benefit was defined as the absolute proportional benefit (LC or OS) of RT alone over no treatment for radical RT indications. For adjuvant indications, RT benefit was the benefit of RT over surgery alone. For radical indications, the survival benefit was estimated to be equal to the survival of patients treated with RT alone (eg, 5-year RT benefit of $20 \%$ if 5 -year OS was $20 \%$ ), unless otherwise stated. LC was defined based on disease control within the radiated field. When these data were unavailable, LC was conservatively estimated based on progression-free survival (PFS). When PFS was unavailable, other survival data was used as described in Table 1.

CRT Benefit: CRT benefit was the incremental benefit (LC or OS) of CRT over RT.

Irreplaceable Benefit of RT: Irreplaceable benefit of RT was defined based on indications where there was no standard-of-practice alternative to RT agreed upon by guidelines. Irreplaceable population LC and OS benefits were estimated.

Population Benefit of RT: Population benefit of RT described the proportion of the population deriving benefit from RT if used according to guidelines compared with no use of RT. This benefit was the proportion of patients benefiting from RT among all patients with malignant brain tumors, rather than an average benefit among patients requiring RT.

\section{Estimation of Incidence of RT Indications}

We used a previously developed RT demand model describing the malignant brain tumor population. ${ }^{1,4}$ This decision tree model described the proportion of patients requiring RT according to evidence-based guidelines. TreeAge Pro 2008 (TreeAge Software, Inc, Williamstown, MA) was used for model development, population benefit estimation, and sensitivity analysis. Each tree described groups of patients, with each additional branch further subdividing patients into progressively smaller subgroups. Each terminal branch described a unique set of conditions defining groups for which RT was or was not indicated. RT was indicated based on better survival, LC, or was quality of life, or a combination of these. The proportion of cases with each RT indication was defined based on epidemiologic data defining the incidence of disease- and patient-related factors. The highest level of epidemiologic data was used, according to a predefined hierarchy. ${ }^{5}$

The RT demand model was adapted in cases for which there were subgroups in a given branch or terminal node with different RT benefits (eg, groupings of age, performance status [PS]) or different RT indications (eg, radical, adjuvant). The same method used to develop the original demand model was applied in these cases.

\section{Systematic Review for RT Indication Benefits}

Systematic review was performed in order to identify the highest level of valid and generalizable evidence defining each RT indication benefit. Data identified from the highest evidence level were used according to the National Health and Medical Research Council (NHMRC) evidence hierarchy. ${ }^{6}$ MEDLINE, all evidence-based medicine sources, and Embase records in Ovid were queried (see supplemental eAppendix 1, available with this article at JNCCN. org). Only studies published in 1990 or later were considered, except where stated otherwise. Literature searches were completed January 2015 through March 2015. Consideration was given to the technical quality of RT used wherever possible (eg, use of MRI for treatment planning, dose, target volume). Population-based SEER data were also queried. To ensure completeness of the search strategy, hand searches of journal article reference lists were performed. In cases when there were multiple sources of the same evidence level, meta-analysis was performed for homogeneous sources.

\section{Statistical Analysis}

A generic inverse-variance meta-analysis was used to determine pooled estimates. Review Manager 5.3 (The Nordic Chochrane Centre, Copenhagen, Denmark) was used. Standard errors were determined for each benefit estimate. Estimates derived from pub- 


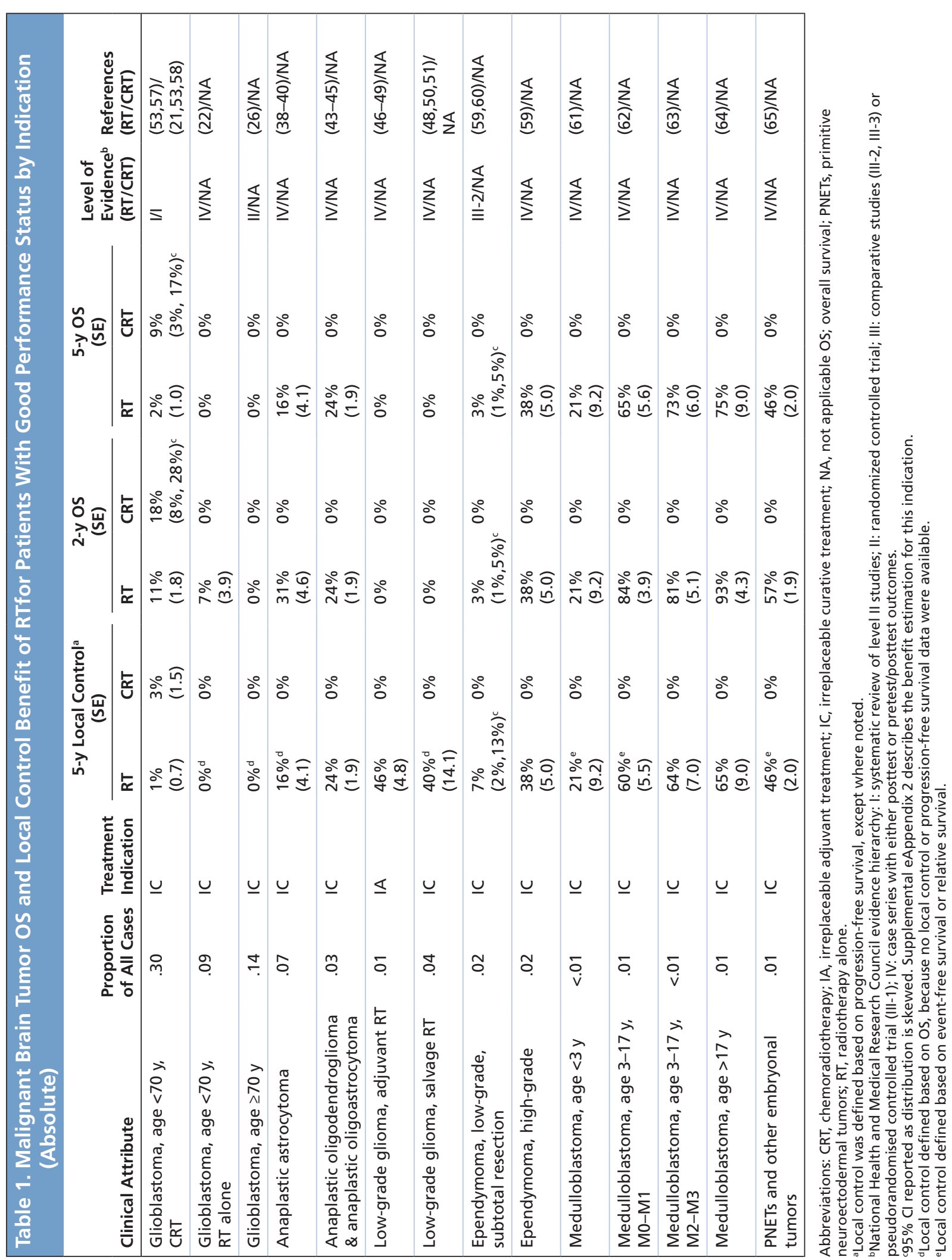


Hanna et al

lished survival curves were used if error estimates were not provided in data sources. Estimates were derived based on Borkowf's hybrid variance estimator, ${ }^{7}$ with the number of events and censored cases estimated using an approach derived from Parmar et $\mathrm{al}^{8}$ and a publicly available spreadsheet published by Tierney et al. ${ }^{9}$

\section{Estimation of the Population Benefit of RT}

The benefit of RT for each indication was associated with the relevant terminal branch in the decision tree. The population benefit of RT was estimated by determining the product of the population proportion with an RT indication and its estimated benefit, and summing all such products.

\section{Sensitivity Analysis}

Deterministic and probabilistic sensitivity analysis was performed. Uncertainties in the incidence of each RT indication and in its associated benefit were considered. Uncertainties in incidence were due to (1) uncertainty regarding epidemiologic data defining the incidence of indications, (2) uncertainty/controversy regarding indications for RT, and (3) uncertainty regarding frequency of optimal use when other equal options existed. Probabilistic multivariate analysis was performed using Monte Carlo simulation. All sources of uncertainty, including statistical uncertainty in each benefit estimate, were simultaneously considered for LC and OS benefits. RT and CRT benefits were separately considered. We performed 10,000 iterations of each simulation. A 95\% CI for each set of iterations was determined based on 2.5th and 97.5th percentile benefit estimates.

\section{Results}

According to guidelines, 3 general uses of curativeintent RT for primary malignant brain tumors were: (1) radical treatment, (2) adjuvant (postoperative) treatment, and (3) treatment of recurrent or progressive disease (Figures 1 and 2). Modifications of the original malignant brain tumor RT demand model are summarized herein.

\section{Radical RT for Good PS Glioblastoma Multiforme} Given uncertainties in management and RT benefit for elderly patients with good PS, the updated model was stratified based on age with a cutoff of 70 years or older, defining 'elderly' (supplemental 1). The small group of patients younger than 70 years with good PS who were unfit for concurrent temozolomide was also considered.

\section{Grade II Glioma}

Given that key trials reported outcomes on lowgrade astrocytoma, low-grade oligodendroglioma, and low-grade oligoastrocytoma together, these were grouped together in the updated model (supplemental eTable 1).

Most guidelines considered use of adjuvant RT a standard option for high-risk grade II glioma, with use of salvage RT for symptomatic, progressive, or recurrent disease after surgery. ${ }^{10-19}$ The EORTC scheme was used to stratify patients (supplemental eTable 1$).{ }^{20}$ Because of uncertainty in the proportion of high-risk patients in the population with RT indications, and optimal risk definition, a sensitivity analysis was performed.

As in the original model, patients identified as having "astrocytoma not otherwise specified" or "other astrocytomas" were considered to most closely represent those with low-grade gliomas (LGGs).

\section{Medulloblastoma and Other Embryonal Tumors} Given its heterogeneity, this group was stratified. Medulloblastoma was the predominant variety. Medulloblastoma was stratified by age given variation in outcome by age group, and also given that studies often reported only on specific ages (supplemental eTable 1).

\section{RT Indication Benefits}

RT benefits are summarized in Table 1. Key findings and decisions made in defining RT indication benefit are summarized herein. LC was often defined based on PFS (Table 1). Considerations for less common histologies are provided in supplemental eAppendix 2 .

\section{Glioblastoma}

Patients Younger Than 70 Years With Good PS: The 2013 Cochrane meta-analysis was used to estimate the survival benefit of CRT for patients with indications. ${ }^{21}$ No data were identified to adequately estimate the benefit of RT for the small group of patients younger than 70 years with good PS treated with RT alone due to contraindications to temozolomide. Out- 


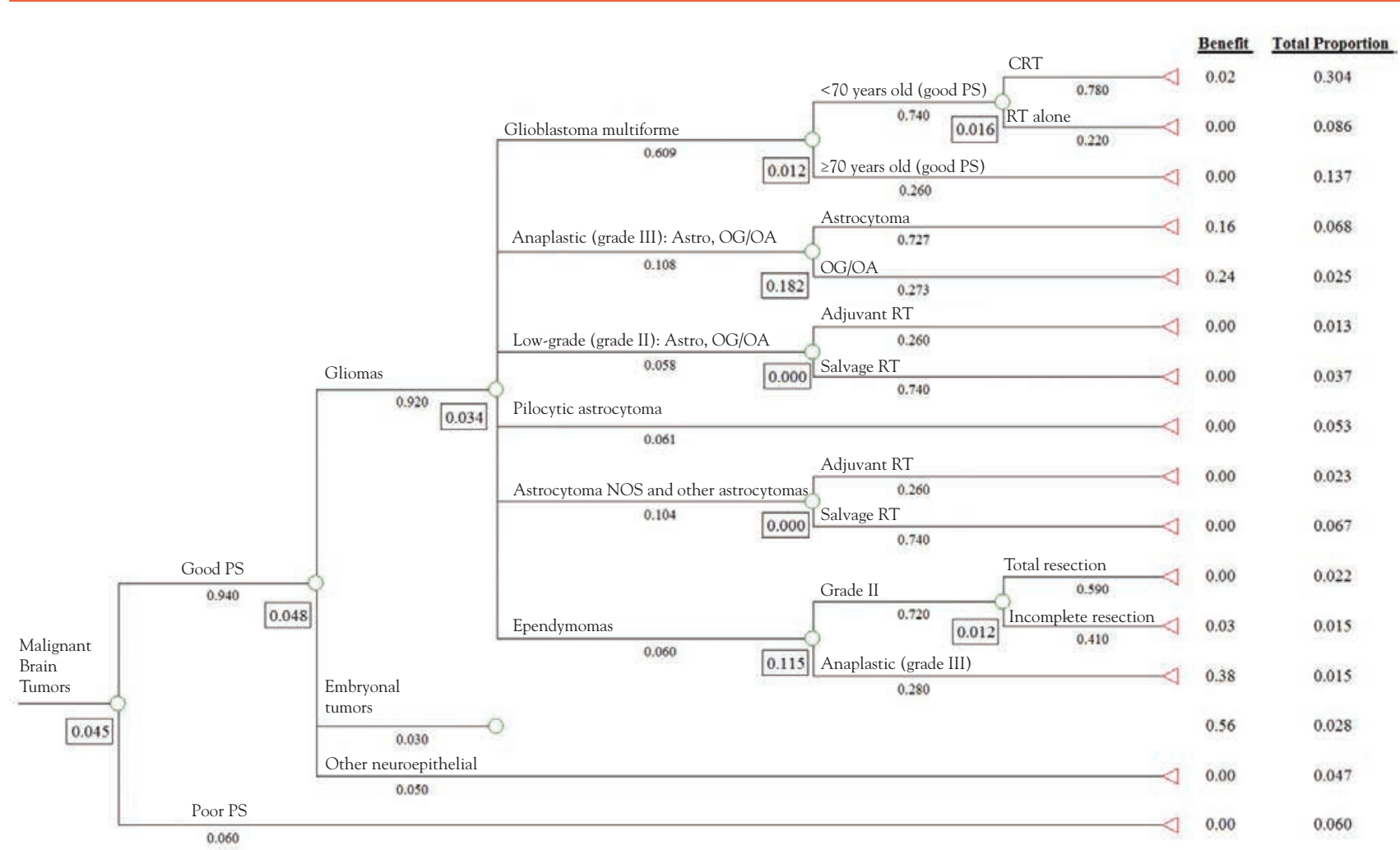

Figure 1. Five-year overall survival benefit of RT alone for malignant brain tumors (embryonal tumor model collapsed; see Figure 2). A similar tree was generated for RT alone local control benefit and for CRT benefits. The value in the rectangular boxes (rollback values) is the population RT benefit for the adjacent stem of the model. The summary benefit estimates for all brain cancer in Table 2 were the rollback values taken from the primary stem (labeled "Malignant Brain Tumors"). The column labeled "Benefit" is the absolute proportional RT benefit associated with each adjacent treatment indication; "Total Proportion" is the proportion of all patients with malignant brain tumors with each treatment indication.

Abbreviations: Astro, astrocytoma; CRT, chemoradiotherapy; NOS, not otherwise specified; OG/OA, oligodendroglioma/oligoastrocytoma; PS, performance status; RT, radiotherapy.

comes for patients younger than 70 years treated with more than 30 Gy but less than 60 Gy of RT alone from a large temozolomide-era case series were used. ${ }^{22}$

Patients Aged 70 Years or Older With Good PS: The literature was searched for relevant studies. Because both age and PS were strong prognostic factors for survival in glioblastoma, strict inclusion criteria were set for both. ${ }^{23-25}$ Studies were included if they reported on patients with a Karnofsky PS (KPS) of 70 or greater and aged 70 years or older, with a sample size of 20 or more (supplemental eAppendix 1). A total of 293 abstracts were identified.

One randomized trial was identified comparing RT and best supportive care. ${ }^{26}$ There were significant benefits to RT, although there were no 2-year survivors in either study arm. Case series similarly reported poor outcomes. ${ }^{24,27-30}$

CRT benefit was also considered. There were no randomized trials, but 9 nonrandomized studies were identified. ${ }^{24,27,30-36}$ Because nearly all series re- ported 2-year survivors with CRT (range, 0\%-24\%), a 2-year survival benefit was considered in sensitivity analysis (Table 1 ). The median study values for 5-year LC and OS with CRT were nil; 5-year benefit was considered nil in the model.

\section{RT for Anaplastic Glioma}

RT outcomes for adult anaplastic astrocytoma (AA), anaplastic oligodendroglioma (AOG), and anaplastic oligoastrocytoma (AOA) were considered (supplemental eAppendix 1). Studies published after 1999 were considered.

For AA, 2 Australian guidelines favored RT alone versus CRT. ${ }^{10,37}$ Studies reporting on patients treated with adjuvant or concurrent chemotherapy were excluded for the RT-alone benefit. An RT dose of 59 to 60 Gy delivered in 1.8 to 2.0 Gy per daily fraction was required and a sample size of 40 or greater. ${ }^{37}$ A total of 3 case series were identified (supplemental eTable 2). ${ }^{38-40}$ OS was estimated based on the 
Hanna et al

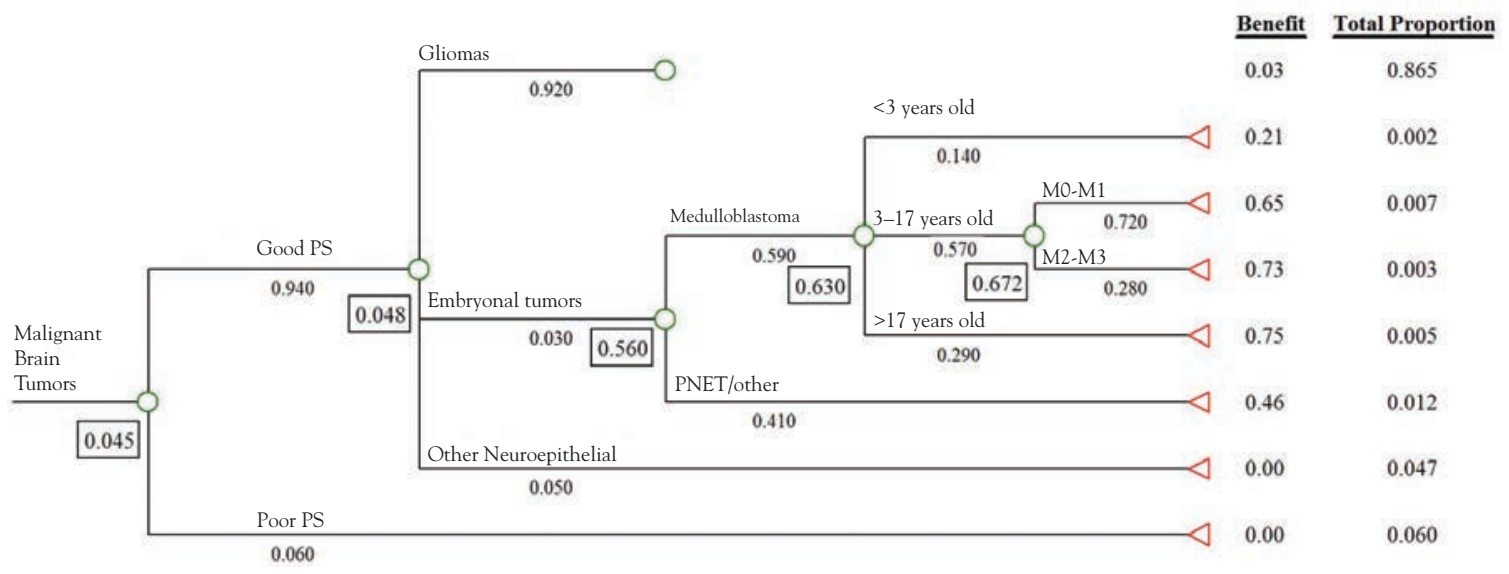

Figure 2. Five-year overall survival benefit of radiotherapy alone for brain cancer (glioma tumor model collapsed; see Figure 1). A similar tree was generated for radiotherapy alone local control benefit and for chemoradiation benefits.

Abbreviations: PNET, primitive neuroectodermal tumors; PS, performance status.

median study value due to data heterogeneity. LC benefit was conservatively estimated to be equal to the 5-year OS benefit, due to the lack of LC data.

AOG and AOA guidelines most often recommended postoperative RT, often in addition to adjuvant chemotherapy. ${ }^{10,12,15,18,37,41,42}$ Given the substantial impact chemotherapy had on survival, the OS benefit of RT was equated to the LC achieved with postoperative RT (supplemental eTable 3). ${ }^{43-45}$

\section{RT for Grade II Glioma}

RT benefit was estimated for the common subtypes: astrocytoma, oligodendroglioma, and oligoastrocytoma. A systematic review was undertaken (supplemental eAppendix 1). Because there was no high-level evidence supporting improved OS with RT for LGG, only LC benefits were estimated. Adult studies published after 1999 reporting on more than 20 patients were considered. Studies reporting on adjuvant RT for unselected patients were excluded, as were data where most patients received adjuvant chemotherapy. Three studies were identified that described postoperative RT LC outcomes for patients with adverse features (supplemental eTable 4). ${ }^{46-49}$ Given the heterogeneity of risk definitions, the median study value of PFS was used to estimate LC benefit. There were limited data on salvage RT outcomes and a case mix of recurrent LGG. ${ }^{48,50,51}$ Sensitivity analysis was performed.

\section{RT Population Benefit for Malignant Brain Tumors}

Of all patients with primary malignant brain tumors, $82 \%$ had indications for adjuvant or curative RT ac- cording to evidence-based guidelines. The magnitude of benefit was based on level I or II evidence in $44 \%$ of all patients. All RT benefits were considered irreplaceable. Overall, in univariate analysis, there was an 11\% 5-year LC benefit (10\% RT, 1\% CRT), $15 \%$ 2-year OS benefit (9\% RT, 6\% CRT), and 8\% 5 -year OS benefit (5\% RT, 3\% CRT) (Figures 1 and 2). Table 2 provides a summary of RT population benefits for malignant brain tumors and common subtypes.

\section{Sensitivity Analysis}

In univariate sensitivity analysis, there were uncertainties in LC benefit for patients with LGG (proportion of patients without RT who experience recurrence and are then recommended to undergo salvage RT: $50 \%-100 \%$; high-risk adjuvant RT LC benefit: 23\%-46\%; salvage RT LC benefit: $22 \%-40 \%$; LGG that are high risk with RT indicated: 9\%-50\%) and ependymoma (grade II total resection LC benefit: $0 \%-7 \%)$. For OS benefit, there was uncertainty about the 2-and 5-year RT benefit for patients with grade II ependymoma who underwent total resection (0\%-3\%) and the 2-year CRT benefit for patients with glioblastoma multiforme (GBM) aged 70 years or older with good PS (0\%-24\%). The model was robust. The population RT LC benefit was most sensitive to the proportion of LGG without prior RT with salvage RT indicated (LC, 7.9\%-10\%), followed by LGG salvage RT benefit uncertainty (LC, 8.1\%-10\%) and LGG high-risk adjuvant RT benefit uncertainty (LC, 9.1\%-10\%); other variables had 
Population Benefit of Radiotherapy: Brain Cancers

little impact on RT LC. OS benefit variation was negligible for RT (eg, 5-year OS, 4.5\%-4.6\%), although CRT 2-year OS varied from 5.5\% to $8.8 \%$ due to uncertainty regarding benefit in elderly patients with GBM.

Considering all of these uncertainties as well as statistical uncertainties in benefit simultaneously in multivariate sensitivity analysis, the 5-year population LC benefit of RT for malignant brain tumors was $8.5 \%$ (95\% CI, 6.9\%-10.3\%), the 2 -year OS benefit was $9.2 \%(95 \% \mathrm{CI}, 7.7 \%-10.6 \%)$, and the 5-year OS benefit was 4.5\% (95\% CI, 3.7\%-5.4\%). The incremental population benefit of CRT over RT for malignant brain tumors was $0.9 \%$ for LC (95\% CI, $0.0 \%-1.8 \%), 7.1 \%$ for 2 -year OS $(95 \%$ CI, $3.6 \%-10.8 \%$ ), and $2.7 \%$ for 5 -year OS (95\% CI, $1.0 \%-5.1 \%)$.

\section{Discussion}

The population benefit of RT was estimated for malignant brain tumors when optimally used according to guidelines, including the incremental benefit of concurrent CRT over RT alone. Estimates were robust in sensitivity analysis. RT alone provided a 5 -year population LC benefit of $8.5 \%$ (95\% CI, $6.9 \%-10.3 \%)$, a 2 -year OS benefit of $9.2 \%$ (95\% CI, $7.7 \%-10.6 \%)$, and a 5 -year OS benefit of $4.5 \%(95 \%$ CI, 3.7\%-5.4\%). The incremental population benefit of CRT over RT was $0.9 \%$ for LC $(95 \%$ CI, 0.0\%$1.8 \%$ ), $7.1 \%$ for 2 -year OS (95\% CI, 3.6\%-10.8\%), and $2.7 \%$ for 5 -year OS (95\% CI, $1.0 \%-5.1 \%)$. It is emphasized that these benefits are factors that may be used with cancer incidence data to estimate the number of patients that could optimally benefit from RT in a population. For example, there are approximately 1,700 malignant brain tumors diagnosed in
Australia annually. ${ }^{52}$ Our findings suggest that each year in Australia, approximately 140 people (95\% CI, 120-180) would derive a 5-year LC benefit and 80 people (95\% CI, 60-90) a 5-year OS benefit from RT alone. CRT was estimated to provide additional benefits, with 20 people deriving a 5 -year LC benefit (95\% CI, 0-30) and 50 people deriving a 5-year OS benefit (95\% CI, 20-90).

There are limitations. Most importantly, the model is limited by the quality of the available epidemiologic and RT outcome data. LC data were limited. For many indications, randomized trials were not available to define RT benefits; for example, involving the use of concurrent chemotherapy with RT for anaplastic (grade III) glioma or elderly patients with GBM and good PS. Uncertainties most often only impact subgroups in the model, limiting their impact on overall population benefit estimates. Where uncertainties were identified, sensitivity analysis was performed. Also, limitations in response assessment due to pseudoprogression may have led to underestimation of the CRT LC benefit for GBM..$^{53,54}$

RT benefits for malignant brain tumors were only considered for LC and OS, and only for specified time points (5-year LC and OS, 2-year OS). RT has other benefits for malignant brain tumors, which include maintaining and improving quality of life, limiting steroid requirements, and reducing the extent of surgery required for LC. RT may also provide shorter-term benefits in both survival and LC not considered in this report. For example, randomized trial evidence supports such benefits for elderly patients with GBM and good PS. ${ }^{26}$ Other modalities are involved in malignant brain tumor management. Distinct benefits of treatments other than RT were not quantified. None of these are limitations fundamental to the method, and should be investigated in further studies.

Table 2. Malignant Brain Tumors OS and Local Control Population Benefit of Radiotherapy (Absolute)

\begin{tabular}{|c|c|c|c|c|c|c|c|c|}
\hline \multirow[b]{2}{*}{ Population of Interest } & \multirow[b]{2}{*}{ Clinical Attribute } & \multirow{2}{*}{$\begin{array}{l}\text { Proportion } \\
\text { of All Cases }\end{array}$} & \multicolumn{2}{|c|}{ 5-y LC } & \multicolumn{2}{|c|}{ 2-y OS } & \multicolumn{2}{|c|}{ 5-y OS } \\
\hline & & & RT & CRT & $\mathbf{R T}$ & CRT & RT & CRT \\
\hline Malignant brain tumors & Whole population & 1.0 & $10 \%$ & $1 \%$ & $9 \%$ & $6 \%$ & $5 \%$ & $3 \%$ \\
\hline Malignant brain, good PS & Glioblastoma & .53 & $1 \%$ & $2 \%$ & $8 \%$ & $10 \%$ & $1 \%$ & $5 \%$ \\
\hline Malignant brain, good PS & Grade III glioma (Astro, OG, OA) & .09 & $18 \%$ & $0 \%$ & $29 \%$ & $0 \%$ & $18 \%$ & $0 \%$ \\
\hline Malignant brain, good PS & Grade II glioma (Astro, OG, OA) & .05 & $42 \%$ & $0 \%$ & $0 \%$ & $0 \%$ & $0 \%$ & $0 \%$ \\
\hline Malignant brain, good PS & Ependymoma & .05 & $13 \%$ & $0 \%$ & $12 \%$ & $0 \%$ & $12 \%$ & $0 \%$ \\
\hline Malignant brain, good PS & Medulloblastoma & .02 & $57 \%$ & $0 \%$ & $77 \%$ & $0 \%$ & $63 \%$ & $0 \%$ \\
\hline
\end{tabular}

Abbreviations: Astro, astrocytoma; CRT, concurrent chemoradiation; LC, local control; OA, oligoastrocytoma; OG, oligodendroglioma; OS, overall survival; PS, performance status; RT, radiotherapy alone. 
Hanna et al

The model used has a number of notable strengths. ${ }^{3}$ It is robust to uncertainties underlying the incidence of RT indications or the associated treatment benefit. The greatest uncertainty in the malignant brain tumor model was for patients with GBM aged 70 years or older with good PS. However, this impacted only the CRT 2-year OS benefit model. A broad range of possibilities were considered (0\%-24\% of patients deriving a 2-year OS benefit). Despite this broad range, the absolute proportional 2-year OS population CRT benefit varied from 5.5\% to $8.8 \%$. In multivariate sensitivity analysis considering this and all other uncertainties, the CRT 2-year OS benefit was still robust (95\% CI, 3.6\%-10.8\%).

Additionally, the model is rapidly adaptable. For example, where epidemiologic parameters have been reported, RT population benefit estimates can be estimated for other settings. Globally, there were 256,213 brain and nervous system tumors diagnosed in 2012 alone..$^{55}$ Adaption of the model to the global cancer population requires consideration. Because the results are reported in terms of absolute proportional benefit, they can be readily adapted when the number of incident malignant brain tumor cases in a population is known according to case mix. Results generated in this way for other cancers were used by the Union for International Cancer Control's Global Task Force on Radiotherapy for Cancer Control to develop an investment case for RT in developing countries. ${ }^{56}$ The model is transparent, with assumptions visible to audit and review. As new data becomes available, the model can be readily updated. For example, if a new CRT benefit were proven in randomized controlled trials, this could be included.

\section{Conclusions}

RT provides important and irreplaceable benefits to many patients in the malignant brain tumor population when optimally used according to guidelines. This includes an important contribution from protocols with radiosensitizing concurrent chemotherapy. The benefit model that was used was robust in sensitivity analysis.

\section{References}

1. 1. Barton $M B$, Jacob S, Shafiq J, et al. Estimating the demand for radiotherapy from the evidence: a review of changes from 2003 to 2012. Radiother Oncol 2014;112:140-144.
2. Hanna TP, Shafiq J, Delaney GP, et al. The population benefit of radiotherapy for cervical cancer: local control and survival estimates for optimally utilized radiotherapy and chemoradiation. Radiother Oncol 2015;114:389-394.

3. Hanna TP, Shafiq J. Estimating the population benefit of radiotherapy: using demand models to estimate achievable cancer outcomes. Clin Oncol (R Coll Radiol) 2015;27:99-106.

4. Barton MB, Jacob S, Shafiq J, et al. Review of optimal radiotherapy utilisation rates. Available at: http://tinyurl.com/pwkua34. Accessed July 26, 2016.

5. Delaney G, Jacob S, Featherstone C, et al. The role of radiotherapy in cancer treatment: estimating optimal utilization from a review of evidencebased clinical guidelines. Cancer 2005;104:129-137.

6. National Health and Medical Research Council. NHMRC levels of evidence and grades for recommendations for guideline developers. Canberra, Australia: National Health and Medical Research Council; 2009. Available at: https://www.nhmrc.gov.au/_files_nhmrc/file/guidelines/ developers/nhmrc_levels_grades_evidence_120423.pdf. Accessed July 26, 2016.

7. Borkowf CB. A simple hybrid variance estimator for the Kaplan-Meier survival function. Stat Med 2005;24:827-851.

8. Parmar MK, Torri V, Stewart L. Extracting summary statistics to perform meta-analyses of the published literature for survival endpoints. Stat Med 1998;17:2815-2834.

9. Tierney JF, Stewart LA, Ghersi D, et al. Practical methods for incorporating summary time-to-event data into meta-analysis. Trials 2007;8:16.

10. Australian Cancer Network Adult Brain Tumour Guidelines Working Party. Clinical Practice Guidelines for the Management of Adult Gliomas: Astrocytomas and Oligodendrogliomas. Sydney, Australia: Cancer Council Australia/Australian Cancer Network/Clinical Oncological Society of Australia Inc.; 2009.

11. eviQ. Radiation Oncology, Brain, Glioma, Adjuvant, Low Grade (LGG) - Adult. Cancer Institute NSW; 2014. Available at: www.eviq.org.au. Accessed July 26, 2016.

12. National Cancer Institute. Adult Central Nervous System Tumors Treatment (PDQ)-Health Professional Version. Available at: http://www. cancer.gov/types/brain/hp/adult-brain-treatment-pdq. Accessed December $30,2014$.

13. Nabors LB, Portnow J, Ammirati M, et al. NCCN Clinical Practice Guidelines in Oncology: Central Nervous System Cancers. Version 1.2016. Accessed July 26, 2016. To view the most recent version of these guidelines, visit NCCN.org.

14. Alberta Health Services. Low-grade astrocytomas and oligodendrogliomas. Clinical Practice Guideline CNS-003 version 3. Available at: http://www. albertahealthservices.ca/assets/info/hp/cancer/if-hp-cancer-guide-cns003low-grade-gliomas.pdf. Accessed July 26, 2016.

15. BC Cancer Agency. Neuro-Oncology. Available at: http://www.bccancer. bc.ca/health-professionals/professional-resources/cancer-managementguidelines/neuro-oncology. Accessed July 26, 2106.

16. Soffietti R, Baumert BG, Bello L, et al. Guidelines on management of low-grade gliomas: report of an EFNS-EANO Task Force. Eur J Neurol 2010;17:1124-1133.

17. Frappaz D, Chinot O, Bataillard A, et al. Summary version of the Standards, Options and Recommendations for the management of adult patients with intracranial glioma (2002). Br J Cancer 2003;89(Suppl 1):S73-83.

18. The Dutch Society for Neuro-Oncology. Neuro-oncology: Gliomas. Version 2.0. Available at: http://www.oncoline.nl/index.php?pagina=/ richtlijn/item/pagina.php\&richtlijn_id=594. Accessed July 26, 2016.

19. Saskatchewan Cancer Agency. Provincial Central Nervous System Cancer Treatment Guidelines. Available at: http://www.saskcancer.ca/CNS\%20 2012\%20c. Accessed July 26, 2016.

20. Pignatti F, van den Bent M, Curran D, et al. Prognostic factors for survival in adult patients with cerebral low-grade glioma. J Clin Oncol 2002;20:2076-2084.

21. Hart MG, Garside R, Rogers G, et al. Temozolomide for high grade glioma. Cochrane Database Syst Rev 2013;4:CD007415.

22. Coate L, McNamara MG, Lwin Z, et al. Glioblastoma treatment in the elderly in the temozolomide therapy era. Can J Neurol Sci 2014;41:357362 .

23. Scott JG, Bauchet L, Fraum TJ, et al. Recursive partitioning analysis of prognostic factors for glioblastoma patients aged 70 years or older. Cancer 2012;118:5595-5600. 
Population Benefit of Radiotherapy: Brain Cancers

24. Barker CA, Chang M, Chou JF, et al. Radiotherapy and concomitant temozolomide may improve survival of elderly patients with glioblastoma. J Neurooncol 2012;109:391-397.

25. Laperriere N, Weller M, Stupp R, et al. Optimal management of elderly patients with glioblastoma. Cancer Treat Rev 2013;39:350-357.

26. Keime-Guibert F, Chinot O, Taillandier L, et al. Radiotherapy for glioblastoma in the elderly. N Engl J Med 2007;356:1527-1535.

27. Reifenberger G, Hentschel B, Felsberg J, et al. Predictive impact of MGMT promoter methylation in glioblastoma of the elderly. Int J Cancer 2012;131:1342-1350.

28. Hoegler DB, Davey P. A prospective study of short course radiotherapy in elderly patients with malignant glioma. J Neurooncol 1997;33:201-204.

29. Idbaih A, Taillibert S, Simon JM, et al. Short course of radiation therapy in elderly patients with glioblastoma multiforme. Cancer Radiothe 2008;12:788-792.

30. Niyazi M, Schwarz SB, Suchorska B, et al. Radiotherapy with and without temozolomide in elderly patients with glioblastoma. Strahlenther Onkol 2012;188:154-159.

31. Minniti G, De Sanctis V, Muni R, et al. Radiotherapy plus concomitant and adjuvant temozolomide for glioblastoma in elderly patients. J Neurooncol 2008;88:97-103.

32. Minniti G, Salvati M, Arcella A, et al. Correlation between O6 methylguanine-DNA methyltransferase and survival in elderly patients with glioblastoma treated with radiotherapy plus concomitant and adjuvant temozolomide. J Neurooncol 2011;102:311-316.

33. Minniti G, Lanzetta G, Scaringi C, et al. Phase II study of short-course radiotherapy plus concomitant and adjuvant temozolomide in elderly patients with glioblastoma. Int J Radiat Oncol Biol Phys 2012;83:93-99.

34. Nguyen LT, Touch S, Nehme-Schuster H, et al. Outcomes in newly diagnosed elderly glioblastoma patients after concomitant temozolomide administration and hypofractionated radiotherapy. Cancers (Basel) 2013;5:1177-1198.

35. Fiorentino A, Balducci M, De Bonis P, et al. Can elderly patients with newly diagnosed glioblastoma be enrolled in radiochemotherapy trials? Am J Clin Oncol 2015;38:23-27.

36. Yin AA, Zhang LH, Cheng JX, et al. Radiotherapy plus concurrent or sequential temozolomide for glioblastoma in the elderly: a meta-analysis. PLoS One 2013;8:e74242.

37. eviQ. Radiation Oncology, Brain, Adjuvant, Grade 3 Glioma High Grade, EBRT Adult. Cancer Institute NSW; 2014. Available at: www.eviq.org.au. Accessed July 26, 2016.

38. Barker CA, Chang M, Beal K, et al. Survival of patients treated with radiation therapy for anaplastic astrocytoma. Radiol Oncol 2014;48:381386.

39. Medical Research Council Brain Tumor Working Party. Randomized trial of procarbazine, lomustine, and vincristine in the adjuvant treatment of high-grade astrocytoma: a Medical Research Council trial. J Clin Oncol 2001;19:509-518.

40. Nagy M, Schulz-Ertner D, Bischof $M$, et al. Long-term outcome of postoperative irradiation in patients with newly diagnosed WHO grade III anaplastic gliomas. Tumori 2009;95:317-324.

41. Alberta Health Services. Anaplastic Astrocytomas and Oligodendrogliomas. Clinical Practice Guideline CNS-002 version 3. Available at: http://www.albertahealthservices.ca/assets/info/hp/cancer/ if-hp-cancer-guide-cns002-anaplastic-gliomas.pdf. Accessed July 26, 2016.

42. Berrocal A, Gil M, Gallego O, et al. SEOM guideline for the treatment of malignant glioma. Clin Transl Oncol 2012;14:545-550.

43. Cairncross G, Wang M, Shaw E, et al. Phase III trial of chemoradiotherapy for anaplastic oligodendroglioma: long-term results of RTOG 9402. J Clin Oncol 2013;31:337-343.

44. Lassman AB, Iwamoto FM, Cloughesy TF, et al. International retrospective study of over 1000 adults with anaplastic oligodendroglial tumors. Neuro Oncol 2011;13:649-659.

45. van den Bent MJ, Brandes AA, Taphoorn MJ, et al. Adjuvant procarbazine, lomustine, and vincristine chemotherapy in newly diagnosed anaplastic oligodendroglioma: long-term follow-up of EORTC brain tumor group study 26951. J Clin Oncol 2013;31:344-350.

46. Buckner JC, Pugh SL, Shaw EG, et al. Phase III study of radiation therapy (RT) with or without procarbazine, CCNU, and vincristine (PCV) in low grade glioma: RTOG 9802 with Alliance, ECOG, and SWOG [abstract]. J Clin Oncol 2014;32(Suppl 1):Abstract 2000.

47. Shaw EG, Wang M, Coons SW, et al. Randomized trial of radiation therapy plus procarbazine, lomustine, and vincristine chemotherapy for supratentorial adult low-grade glioma: initial results of RTOG 9802. J Clin Oncol 2012;30:3065-3070

48. Yeh SA, Ho JT, Lui CC, et al. Treatment outcomes and prognostic factors in patients with supratentorial low-grade gliomas. Br J Radiol 2005; 78:230-235.

49. Youland RS, Brown PD, Giannini C, et al. Adult low-grade glioma: 19. year experience at a single institution. Am J Clin Oncol 2013;36:612-619.

50. Hanzely Z, Polgar C, Fodor J, et al. Role of early radiotherapy in the treatment of supratentorial WHO Grade II astrocytomas: long-term results of 97 patients. J Neurooncol 2003;63:305-312

51. Baumert BG, Mason WP, Ryan G, et al. Temozolomide chemotherapy versus radiotherapy in molecularly characterized (1p loss) low-grade glioma: a randomized phase III intergroup study by the EORTC/NCICCTG/TROG/MRC-CTU (EORTC 22033-26033) [abstract]. J Clin Oncol 2013;31(Suppl):Abstract 2007

52. Australian Institute of Health and Welfare. Australian Cancer Incidence and Mortality (ACIM) Books. Brain Cancer (ICD10 C71). Available at: http://www.aihw.gov.au/acim-books/. Accessed October 17, 2015.

53. Stupp R, Hegi ME, Mason WP, et al. Effects of radiotherapy with concomitant and adjuvant temozolomide versus radiotherapy alone on survival in glioblastoma in a randomised phase III study: 5 -year analysis of the EORTC-NCIC trial. Lancet Oncol 2009;10:459-466.

54. Brandes AA, Franceschi E, Tosoni A, et al. MGMT promoter methylation status can predict the incidence and outcome of pseudoprogression after concomitant radiochemotherapy in newly diagnosed glioblastoma patients. J Clin Oncol 2008;26:2192-2197.

55. Ferlay J, Soerjomataram I, Ervik M, et al. GLOBOCAN 2012 v1.0, Cancer Incidence and Mortality Worldwide: IARC CancerBase No. 11 [Internet]. Lyon, France: International Agency for Research on Cancer; 2013. Available at: http://globocan.iarc.fr. Accessed October 19, 2015.

56. Atun R, Jaffray DA, Barton $\mathrm{MB}$, et al. Expanding global access to radiotherapy. Lancet Oncol 2015;16:1153-1186.

57. Laperriere N, Zuraw L, Cairncross G, et al. Radiotherapy for newly diagnosed malignant glioma in adults: a systematic review. Radiother Oncol 2002;64:259-273.

58. Athanassiou H, Synodinou M, Maragoudakis E, et al. Randomized phase II study of temozolomide and radiotherapy compared with radiotherapy alone in newly diagnosed glioblastoma multiforme. J Clin Oncol 2005;23:2372_ 2377.

59. Korshunov A, Golanov A, Sycheva R, et al. The histologic grade is a main prognostic factor for patients with intracranial ependymomas treated in the microneurosurgical era: an analysis of 258 patients. Cancer 2004;100:1230-1237.

60. Xavier A, Costa L, Bredlau AL. Survival in a large database of unselected patients with ependymoma: effects of age, tumor location and treatment patterns. Pediatr Blood Cancer 2013;60:S69

61. von Bueren AO, von Hoff $K$, Pietsch $T$, et al. Treatment of young children with localized medulloblastoma by chemotherapy alone: results of the prospective, multicenter trial HIT 2000 confirming the prognostic impact of histology. Neuro Oncol 2011;13:669-679.

62. Taylor RE, Bailey CC, Robinson $\mathrm{K}$, et al. Results of a randomized study of preradiation chemotherapy versus radiotherapy alone for nonmetastatic medulloblastoma: The International Society of Paediatric Oncology/ United Kingdom Children's Cancer Study Group PNET-3 study. J Clin Oncol 2003;21:1581-1591.

63. Jakacki RI, Burger PC, Zhou $T$, et al. Outcome of children with metastatic medulloblastoma treated with carboplatin during craniospinal radiotherapy: a Children's Oncology Group phase I/II study. J Clin Oncol 2012;30:2648-2653

64. Brandes AA, Ermani M, Amista P, et al. The treatment of adults with medulloblastoma: a prospective study. Int J Radiat Oncol Biol Phys 2003;57:755-761.

65. Ostrom QT, Gittleman H, Liao P, et al. CBTRUS statistical report: primary brain and central nervous system tumors diagnosed in the United States in 2007-2011. Neuro Oncol 2014;16(Suppl 4:)iv1-63. 